\title{
Crystal structure of $(R)-N, N^{\prime}, N^{\prime}, N^{\prime \prime}, N^{\prime \prime}$-pentamethyl- $N$-(1-phenylethyl)- guanidinium iodide, $\left[\mathrm{C}_{14} \mathrm{H}_{24} \mathrm{~N}_{3}\right] \mathrm{I}$
}

\author{
Wolfgang Frey, Thomas Orbegozo, Dietrich Spitzner and Volker Jäger ${ }^{*}$ \\ Universität Stuttgart, Institut für Organische Chemie, Pfaffenwaldring 55, 70569 Stuttgart, Germany
}

Received February 24, 2009, accepted and available on-line March 11, 2009; CCDC no. 1267/2570

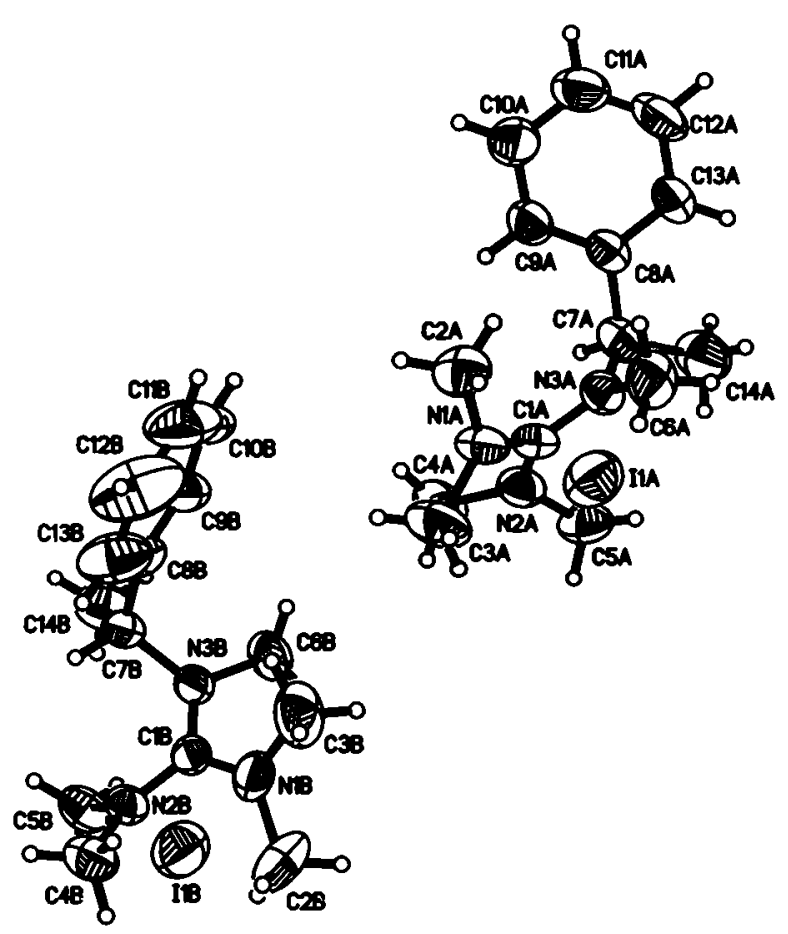

Abstract

$\mathrm{C}_{14} \mathrm{H}_{24} \mathrm{IN}_{3}$, monoclinic, $P 12_{1} 1$ (no. 4), $a=10.599(3) \AA$,

$b=10.737(4) \AA, c=14.970(5) \AA, \beta=90.02(3)^{\circ}$,

$V=1703.6 \AA^{3}, Z=4, R_{g d}(F)=0.054, w R_{\text {ref }}\left(F^{2}\right)=0.149$,

$T=293 \mathrm{~K}$.

\section{Source of material}

The title compound was prepared by methylation of $(R)$ $N, N, N^{\prime}, N^{\prime}$-tetramethyl- $N$-(1-phenylethyl)guanidine [1] with methyl iodide. The crude product was recrystallized from acetonitrile/diethyl ether, m.p. $145-146^{\circ} \mathrm{C}$. The enantiomerically pure compound has $[\alpha]_{\mathrm{D}}^{20}=-14.3$ (c = 1.00 , acetonitrile).

\section{Experimental details}

The $\beta$ angle of the unit cell is $90.02(3)^{\circ}$, which is close to the orthorhombic crystal system. All attempts solving the structure in the orthorhombic crystal system failed. Checking the Laue symmetry of some reflections had confirmed the monoclinic system in this case. $\mathrm{H}$ atoms were located on difference fourier map, but refined with fixed individual displacement parameters using a riding model with $d(\mathrm{C}-\mathrm{H})$ ranging from 0.93 to $0.98 \AA$. In addition, the methyl groups were allowed to rotate but not to tip.

\footnotetext{
* Correspondence author (e-mail: jager.ioc@oc.uni-stuttgart.de)
}

\section{Discussion}

The title compound crystallizes with two independent molecules in the asymmetric unit. The absolute configuration, as known from the starting material, $(R)-N, N, N^{\prime}, N^{\prime}$-tetramethyl- $N$-(1phenylethyl)guanidine, was confirmed by the absolute structure determination by $X$-ray data indicated by the Flack parameter of $x$ $=0.00(3)$. The guanidinium ion is showing a planar conformation. In contrast, the methyl groups attached to the nitrogen atoms are forced out-of-plane. Both conformers (molecule $A$ and molecule B) differ slightly in this distortion characterized by the dihedral angles between N1-C2-C3 and N2-C4-C5 of $58.4(5)^{\circ}$ [A] and 58.2(6) ${ }^{\circ}$ [B]. Between N2-C4-C5 and N3-C6-C7 the dihedral angles are $71.8(5)^{\circ}[\mathrm{A}]$ and $68.2(5)^{\circ}[\mathrm{B}]$. The dihedral angles between $\mathrm{N} 3-\mathrm{C} 6-\mathrm{C} 7$ and $\mathrm{N} 1-\mathrm{C} 2-\mathrm{C} 3$ are $60.5(5)^{\circ}[\mathrm{A}]$ and $64.7(5)^{\circ}[B]$.

W.

Table 1. Data collection and handling.

\section{Crystal:}

Wavelength:

$\mu$ :

Diffractometer, scan mode:

20 $\theta_{\max }$ :

$N(h k l)_{\text {measured, }} N(h k l)_{\text {unique: }}$ Criterion for $l_{\mathrm{obs}}, N(h k l)_{\mathrm{gt}}$ :

$N($ param) refined:

Programs: colorless plates,

size $0.35 \times 0.70 \times 1.20 \mathrm{~mm}$

Mo $K_{\alpha}$ radiation $(0.71073 \AA)$

$18.70 \mathrm{~cm}^{-1}$

Nicolet P3, Wyckoff scan

$60^{\circ}$

10696, 9905

$I_{\text {obs }}>2 \sigma\left(I_{\text {obs }}\right), 7327$

338

SHELXS-97 [3], SHELXL-97 [4], SHEL XTL-Plus [5]
Table 2. Atomic coordinates and displacement parameters (in $\AA^{2}$ ).

\begin{tabular}{lllrrr}
\hline Atom & Site & \multicolumn{1}{l}{$\boldsymbol{x}$} & \multicolumn{1}{c}{$\boldsymbol{y}$} & \multicolumn{1}{l}{$\boldsymbol{U}_{\text {iso }}$} \\
\hline H(2A1) & $2 a$ & 1.1213 & 0.0432 & -0.0017 & 0.102 \\
H(2A2) & $2 a$ & 1.0002 & 0.0273 & 0.0573 & 0.102 \\
H(2A3) & $2 a$ & 1.0966 & -0.0827 & 0.0483 & 0.102 \\
H(3A1) & $2 a$ & 1.1290 & -0.0862 & 0.2169 & 0.130 \\
H(3A2) & $2 a$ & 1.0239 & 0.0164 & 0.2213 & 0.130 \\
H(3A3) & $2 a$ & 1.1571 & 0.0418 & 0.2632 & 0.130 \\
H(4A1) & $2 a$ & 1.3691 & 0.0025 & 0.2760 & 0.122 \\
H(4A2) & $2 a$ & 1.4858 & 0.0124 & 0.2120 & 0.122 \\
H(4A3) & $2 a$ & 1.3626 & -0.0547 & 0.1798 & 0.122 \\
H(5A1) & $2 a$ & 1.3600 & 0.3126 & 0.2011 & 0.102 \\
H(5A2) & $2 a$ & 1.4919 & 0.2476 & 0.1924 & 0.102 \\
H(5A3) & $2 a$ & 1.4136 & 0.2337 & 0.2807 & 0.102 \\
H(6A1) & $2 a$ & 1.1009 & 0.2532 & -0.0195 & 0.110 \\
H(6A2) & $2 a$ & 1.1411 & 0.3736 & 0.0325 & 0.110 \\
H(6A3) & $2 a$ & 1.0665 & 0.2679 & 0.0818 & 0.110 \\
H(7A) & $2 a$ & 1.4312 & 0.1997 & 0.0273 & 0.069 \\
H(9A) & $2 a$ & 1.3522 & 0.0303 & -0.0663 & 0.073 \\
H(10A) & $2 a$ & 1.2882 & -0.0377 & -0.2063 & 0.088 \\
H(11A) & $2 a$ & 1.2342 & 0.1058 & -0.3132 & 0.092 \\
H(12A) & $2 a$ & 1.2335 & 0.3139 & -0.2818 & 0.099 \\
\hline & & & & & \\
\hline
\end{tabular}


Table 2. Continued.

\begin{tabular}{llllll}
\hline Atom & Site & $x$ & $y$ & $z$ & $U_{\text {iso }}$ \\
\hline H(13A) & $2 a$ & 1.2997 & 0.3839 & -0.1410 & 0.083 \\
H(14A) & $2 a$ & 1.3395 & 0.4440 & -0.0035 & 0.134 \\
H(14B) & $2 a$ & 1.4783 & 0.4025 & -0.0241 & 0.134 \\
H(14C) & $2 a$ & 1.4301 & 0.4063 & 0.0748 & 0.134 \\
H(2B1) & $2 a$ & 1.0501 & -0.1855 & 0.6106 & 0.150 \\
H(2B2) & $2 a$ & 1.1423 & -0.0737 & 0.5939 & 0.150 \\
H(2B3) & $2 a$ & 1.1889 & -0.1913 & 0.6465 & 0.150 \\
H(3B1) & $2 a$ & 1.1518 & -0.0811 & 0.4280 & 0.141 \\
H(3B2) & $2 a$ & 1.0296 & -0.1604 & 0.4433 & 0.141 \\
H(3B3) & $2 a$ & 1.1398 & -0.2136 & 0.3848 & 0.141 \\
H(4B1) & $2 a$ & 1.0606 & -0.4088 & 0.5859 & 0.102 \\
H(4B2) & $2 a$ & 1.1306 & -0.4086 & 0.6782 & 0.102 \\
H(4B3) & $2 a$ & 1.1262 & -0.5304 & 0.6205 & 0.102 \\
H(5B1) & $2 a$ & 1.3564 & -0.5570 & 0.5767 & 0.095 \\
\hline
\end{tabular}

Table 2. Continued.

\begin{tabular}{llllll}
\hline Atom & Site & $x$ & $y$ & $z$ & $U_{\text {iso }}$ \\
\hline H(5B2) & $2 a$ & 1.3610 & -0.4810 & 0.6661 & 0.095 \\
H(5B3) & $2 a$ & 1.4340 & -0.4329 & 0.5818 & 0.095 \\
H(6B1) & $2 a$ & 1.3829 & -0.1481 & 0.4464 & 0.126 \\
H(6B2) & $2 a$ & 1.3751 & -0.2032 & 0.3496 & 0.126 \\
H(6B3) & $2 a$ & 1.4917 & -0.2333 & 0.4104 & 0.126 \\
H(7B) & $2 a$ & 1.3039 & -0.5114 & 0.4266 & 0.062 \\
H(9B) & $2 a$ & 1.4322 & -0.3628 & 0.2355 & 0.083 \\
H(10B) & $2 a$ & 1.3224 & -0.3241 & 0.1018 & 0.106 \\
H(11B) & $2 a$ & 1.1170 & -0.3693 & 0.0934 & 0.137 \\
H(12B) & $2 a$ & 1.0093 & -0.4543 & 0.2090 & 0.159 \\
H(13B) & $2 a$ & 1.1163 & -0.4964 & 0.3448 & 0.116 \\
H(14D) & $2 a$ & 1.5329 & -0.4334 & 0.3495 & 0.117 \\
H(14E) & $2 a$ & 1.4886 & -0.5720 & 0.3595 & 0.117 \\
H(14F) & $2 a$ & 1.5235 & -0.4941 & 0.4445 & 0.117 \\
\hline
\end{tabular}

Table 3. Atomic coordinates and displacement parameters (in $\AA^{2}$ ).

\begin{tabular}{|c|c|c|c|c|c|c|c|c|c|c|}
\hline Atom & Site & $x$ & $y$ & $z$ & $U_{11}$ & $U_{22}$ & $U_{33}$ & $U_{12}$ & $U_{13}$ & $U_{23}$ \\
\hline $\mathbf{I}(\mathbf{A})$ & $2 a$ & $0.76258(4)$ & $0.18461(4)$ & $0.12610(3)$ & $0.0813(2)$ & $0.0743(3)$ & $0.0730(2)$ & $0.0057(2)$ & $0.0086(2)$ & $-0.0128(2)$ \\
\hline I(1B) & $2 a$ & $0.76360(4)$ & $-0.30102(4)$ & $0.53683(3)$ & $0.0910(3)$ & $0.0673(2)$ & $0.0658(2)$ & $0.0120(2)$ & $-0.0117(2)$ & $-0.0065(2)$ \\
\hline$C(1 A)$ & $2 a$ & $1.2499(4)$ & $0.1350(5)$ & $0.1234(3)$ & $0.045(2)$ & $0.059(3)$ & $0.032(2)$ & $0.002(2)$ & $-0.001(2)$ & $-0.002(2)$ \\
\hline$N(1 A)$ & $2 a$ & $1.1568(4)$ & $0.0528(5)$ & $0.1292(3)$ & $0.060(2)$ & $0.073(3)$ & $0.036(2)$ & $-0.011(2)$ & $0.003(2)$ & $-0.001(2)$ \\
\hline $\mathbf{N}(2 A)$ & $2 a$ & $1.3477(4)$ & $0.1296(4)$ & $0.1809(3)$ & $0.059(2)$ & $0.064(3)$ & $0.042(2)$ & $0.003(2)$ & $-0.008(2)$ & $0.003(2)$ \\
\hline$C(2 A)$ & $2 a$ & $1.0878(5)$ & $0.0062(7)$ & $0.0517(4)$ & $0.060(3)$ & $0.080(4)$ & $0.064(3)$ & $-0.016(3)$ & $-0.008(2)$ & $-0.008(3)$ \\
\hline$N(3 A)$ & $2 a$ & $1.2478(3)$ & $0.2258(4)$ & $0.0618(3)$ & $0.049(2)$ & $0.055(2)$ & $0.049(2)$ & $0.004(2)$ & $-0.003(2)$ & $0.005(2)$ \\
\hline$C(3 A)$ & $2 a$ & $1.1129(7)$ & $0.0017(8)$ & $0.2151(5)$ & $0.093(5)$ & $0.112(6)$ & $0.055(3)$ & $-0.025(4)$ & $0.020(3)$ & $0.016(4)$ \\
\hline$C(4 A)$ & $2 a$ & $1.3953(7)$ & $0.0126(7)$ & $0.2150(5)$ & $0.095(5)$ & $0.089(5)$ & $0.061(4)$ & $0.017(4)$ & $-0.018(3)$ & $0.022(3)$ \\
\hline $\mathrm{C}(5 \mathrm{~A})$ & $2 a$ & $1.4085(6)$ & $0.2403(6)$ & $0.2168(4)$ & $0.073(3)$ & $0.085(4)$ & $0.045(3)$ & $-0.013(3)$ & $-0.008(2)$ & $-0.014(3)$ \\
\hline$C(6 A)$ & $2 a$ & $1.1289(6)$ & $0.2852(7)$ & $0.0370(5)$ & $0.066(3)$ & $0.069(4)$ & $0.084(4)$ & $0.019(3)$ & $-0.014(3)$ & $0.003(3)$ \\
\hline$C(7 A)$ & $2 a$ & $1.3626(5)$ & $0.2531(5)$ & $0.0059(4)$ & $0.055(3)$ & $0.065(3)$ & $0.053(3)$ & $-0.009(2)$ & $-0.001(2)$ & $0.013(2)$ \\
\hline $\mathrm{C}(8 \mathrm{~A})$ & $2 a$ & $1.3307(4)$ & $0.2131(5)$ & $-0.0891(3)$ & $0.060(2)$ & $0.056(3)$ & $0.046(2)$ & $-0.001(2)$ & $0.004(2)$ & $0.011(2)$ \\
\hline$C(9 A)$ & $2 a$ & $1.3283(6)$ & $0.0881(6)$ & $-0.1094(4)$ & $0.073(3)$ & $0.057(3)$ & $0.052(3)$ & $0.009(2)$ & $0.014(2)$ & $0.010(2)$ \\
\hline$C(10 A)$ & $2 a$ & $1.2907(7)$ & $0.0469(7)$ & $-0.1934(5)$ & $0.090(4)$ & $0.067(4)$ & $0.063(4)$ & $-0.003(3)$ & $0.024(3)$ & $-0.002(3)$ \\
\hline$C(11 A)$ & $2 a$ & $1.2577(8)$ & $0.1328(8)$ & $-0.2566(5)$ & $0.092(5)$ & $0.090(5)$ & $0.048(3)$ & $-0.016(4)$ & $0.007(3)$ & $0.003(3)$ \\
\hline$C(12 A)$ & $2 a$ & $1.2584(8)$ & $0.2570(9)$ & $-0.2383(5)$ & $0.106(5)$ & $0.091(5)$ & $0.050(4)$ & $-0.012(4)$ & $-0.004(3)$ & $0.029(3)$ \\
\hline$C(13 A)$ & $2 a$ & $1.2970(7)$ & $0.2991(6)$ & $-0.1535(4)$ & $0.100(4)$ & $0.053(3)$ & $0.054(3)$ & $-0.007(3)$ & $-0.004(3)$ & $0.015(3)$ \\
\hline$C(14 A)$ & $2 a$ & $1.4068(8)$ & $0.3894(8)$ & $0.0140(5)$ & $0.113(5)$ & $0.085(5)$ & $0.069(4)$ & $-0.039(4)$ & $-0.011(4)$ & $0.008(4)$ \\
\hline $\mathrm{C}(\mathbf{1 B})$ & $2 a$ & $1.2520(4)$ & $-0.3172(5)$ & $0.5114(3)$ & $0.061(2)$ & $0.040(2)$ & $0.038(2)$ & $-0.008(2)$ & $-0.012(2)$ & $-0.002(2)$ \\
\hline $\mathbf{N}(\mathbf{B})$ & $2 a$ & $1.1765(5)$ & $-0.2192(4)$ & $0.5157(4)$ & $0.089(3)$ & $0.041(2)$ & $0.063(3)$ & $0.009(2)$ & $-0.009(2)$ & $-0.004(2)$ \\
\hline $\mathbf{N}(2 B)$ & $2 a$ & $1.2476(4)$ & $-0.4046(4)$ & $0.5742(3)$ & $0.057(2)$ & $0.052(2)$ & $0.037(2)$ & $-0.003(2)$ & $-0.006(2)$ & $0.004(2)$ \\
\hline $\mathrm{C}(2 \mathrm{~B})$ & $2 a$ & $1.1360(9)$ & $-0.1627(8)$ & $0.5987(7)$ & $0.120(7)$ & $0.072(5)$ & $0.108(7)$ & $0.015(4)$ & $-0.001(5)$ & $-0.043(5)$ \\
\hline$C(3 B)$ & $2 a$ & $1.1195(8)$ & $-0.1638(7)$ & $0.4361(6)$ & $0.121(6)$ & $0.064(4)$ & $0.098(6)$ & $0.013(4)$ & $-0.036(5)$ & $0.015(4)$ \\
\hline N(3B) & $2 a$ & $1.3370(4)$ & $-0.3287(4)$ & $0.4445(2)$ & $0.067(2)$ & $0.046(2)$ & $0.040(2)$ & $-0.015(2)$ & $-0.003(1)$ & $0.001(2)$ \\
\hline$C(4 B)$ & $2 a$ & $1.1314(6)$ & $-0.4412(7)$ & $0.6184(5)$ & $0.069(3)$ & $0.082(4)$ & $0.053(3)$ & $-0.012(3)$ & $0.007(3)$ & $0.007(3)$ \\
\hline $\mathrm{C}(5 \mathrm{~B})$ & $2 a$ & $1.3594(5)$ & $-0.4750(6)$ & $0.6021(4)$ & $0.062(3)$ & $0.075(4)$ & $0.054(3)$ & $0.005(3)$ & $-0.008(2)$ & $0.019(3)$ \\
\hline$C(6 B)$ & $2 a$ & $1.4023(8)$ & $-0.2189(6)$ & $0.4098(5)$ & $0.125(6)$ & $0.061(3)$ & $0.066(4)$ & $-0.049(4)$ & $0.002(4)$ & $0.007(3)$ \\
\hline $\mathrm{C}(7 \mathrm{~B})$ & $2 a$ & $1.3502(5)$ & $-0.4472(5)$ & $0.3936(3)$ & $0.063(3)$ & $0.053(3)$ & $0.038(2)$ & $-0.008(2)$ & $0.003(2)$ & $0.001(2)$ \\
\hline $\mathrm{C}(\mathbf{8 B})$ & $2 a$ & $1.2842(5)$ & $-0.4307(6)$ & $0.3042(4)$ & $0.059(3)$ & $0.067(3)$ & $0.050(3)$ & $0.001(2)$ & $-0.001(2)$ & $-0.018(2)$ \\
\hline $\mathrm{C}(9 \mathrm{~B})$ & $2 a$ & $1.3466(6)$ & $-0.3811(7)$ & $0.2314(4)$ & $0.081(4)$ & $0.081(4)$ & $0.046(3)$ & $-0.001(3)$ & $-0.003(2)$ & $-0.003(3)$ \\
\hline $\mathrm{C}(10 \mathrm{~B})$ & $2 a$ & $1.2809(9)$ & $-0.3581(8)$ & $0.1508(4)$ & $0.125(6)$ & $0.096(5)$ & $0.043(3)$ & $0.022(4)$ & $-0.010(3)$ & $-0.008(3)$ \\
\hline$C(11 B)$ & $2 a$ & $1.160(1)$ & $-0.386(1)$ & $0.1464(6)$ & $0.136(8)$ & $0.149(9)$ & $0.058(4)$ & $0.035(7)$ & $-0.030(5)$ & $-0.022(5)$ \\
\hline$C(12 B)$ & $2 a$ & $1.0946(8)$ & $-0.436(1)$ & $0.2151(8)$ & $0.070(4)$ & $0.21(1)$ & $0.118(8)$ & $0.005(6)$ & $-0.033(5)$ & $-0.065(8)$ \\
\hline$C(13 B)$ & $2 a$ & $1.1589(7)$ & $-0.461(1)$ & $0.2970(5)$ & $0.075(4)$ & $0.140(8)$ & $0.075(5)$ & $-0.015(4)$ & $-0.003(3)$ & $-0.030(5)$ \\
\hline$C(14 B)$ & $2 a$ & $1.4863(6)$ & $-0.4907(8)$ & $0.3861(4)$ & $0.071(4)$ & $0.103(5)$ & $0.061(4)$ & $0.008(3)$ & $-0.003(3)$ & $0.006(3)$ \\
\hline
\end{tabular}

\section{References}

1. Spitzner, D.; Jäger, V.: Unpublished results, 2007/2008.

2. Orbegozo, T.: Diplomarbeit, Universität Stuttgart 2006.

3. Sheldrick, G. M.: SHELXS-97. Program for the Solution of Crystal Structures. University of Göttingen, Germany 1997.
4. Sheldrick, G. M.: SHELXL-97. Program for the Refinement of Crystal Structures. University of Göttingen, Germany 1997.

5. Sheldrick, G. M.: SHELXTL-Plus. Structure Determination Software Suite. Release 4.1. Siemens Analytical Systems Inc., Madison, Wisconsin, USA 1991 